\title{
Anti-proteinase 3 antineutrophil cytoplasmic antibody reflects disease activity and predicts the response to steroid therapy in ulcerative colitis
}

Yuki Aoyama', Tomoki Inaba', Sakuma Takahashi ${ }^{*} \mathbb{C}^{1}$, Hisae Yasuhara², Sakiko Hiraoka ${ }^{3}$, Takeshi Morimoto ${ }^{4}$, Hugh Shunsuke Colvin ${ }^{1}$, Masaki Wato ${ }^{1}$, Midori Ando ${ }^{5}$, Satoko Nakamura ${ }^{5}$, Koichi Mizobuchi ${ }^{5}$ and Hiroyuki Okada ${ }^{3}$

\begin{abstract}
Background: Serum anti-proteinase 3 antineutrophil cytoplasmic antibody (PR3-ANCA) is a disease-specific antibody against granulomatosis with polyangiitis. PR3-ANCA is a useful serological marker for disease severity in ulcerative colitis (UC). The purpose of this study was to investigate whether PR3-ANCA levels could also predict the success of induction therapy and to compare its performance against other markers, including serum CRP and fecal hemoglobin.

Methods: This was a multicenter retrospective study. In total, 159 patients with active-phase UC underwent colonoscopy. Disease activity was measured using the Mayo endoscopic subscore (MES). PR3-ANCA positivity and the response to induction therapy, either 5-aminosalicylic acid or steroid, were assessed. PR3-ANCA, CRP, and fecal hemoglobin were measured during the active phase, and during clinical remission.

Results: Eighty-five (53.5\%) of 159 patients with active UC were positive for PR3-ANCA. PR3-ANCA titers were significantly higher in the group of patients with MES 3 compared to patients with MES $1(P=0.002)$ or MES $2(P=0.035)$. Steroid therapy was administered to 56 patients with a median partial Mayo score of 7 (5-9), which is equivalent to moderate-to-severe disease activity. PR3-ANCA positivity of non-responders to steroid therapy was significantly higher than that of responders ( $71.9 \% \mathrm{vs}, 41.7 \%, P=0.030)$, whereas CRP and fecal hemoglobin were not predictive of steroid response. Multivariate analysis demonstrated that PR3-ANCA positivity was associated with non-response to steroid therapy (odds ratio $5.19 ; 95 \%$ confidence interval, $1.54-17.5 ; P=0.008$ ). Of the 37 patients treated to clinical remission who were also positive for PR3-ANCA during the active phase, 27 had an MES of $\geq 1$, and 10 patients had an MES of 0 . In clinical remission, the proportion of patients with MES 0 in 17 patients whose PR3-ANCA became negative was significantly higher than that in 20 patients whose PR3-ANCA remained positive $(47.1 \%$ vs. $10.0 \%, P=0.023)$.
\end{abstract}

Conclusions: PR3-ANCA not only serves as a marker of disease activity, but also predicts the failure of steroid therapy in moderate-to-severe UC.

\footnotetext{
*Correspondence: saqi@yahoo.co.jp

${ }^{1}$ Department of Gastroenterology, Kagawa Prefectural Central Hospital,

1-2-1 Asahi-machi, Takamatsu, Kagawa 760-8557, Japan

Full list of author information is available at the end of the article
} original author(s) and the source, provide a link to the Creative Commons licence, and indicate if changes were made. The images or other third party material in this article are included in the article's Creative Commons licence, unless indicated otherwise in a credit line to the material. If material is not included in the article's Creative Commons licence and your intended use is not permitted by statutory regulation or exceeds the permitted use, you will need to obtain permission directly from the copyright holder. To view a copy of this licence, visit http://creativecommons.org/licenses/by/4.0/. The Creative Commons Public Domain Dedication waiver (http://creativecommons.org/publicdomain/zero/1.0/) applies to the data made available in this article, unless otherwise stated in a credit line to the data. 
Trial registration: This study was retrospectively registered in the UMIN Clinical Trials Registry System (000039174) on January 16, 2020.

Keywords: Ulcerative colitis, Biomarkers, Antineutrophil cytoplasmic antibody, Steroids, Refractory, Colonoscopy

\section{Background}

Ulcerative colitis (UC) is a chronic refractory inflammatory bowel disease of unknown cause [1], and its treatment is determined according to disease extent and activity. The first-line therapy for patients with mild-tomoderate activity is 5-aminosalicylic acid (5-ASA). Corticosteroids are used for patients who are refractory to 5-ASA or who have moderate-to-severe activity. However, approximately half of patients are resistant to or dependent on corticosteroids [2]. Several predictors of non-response to steroids, including lower hemoglobin level, partial Mayo score [3], and disease duration [4], have been reported. However, none of these markers are capable of fully predicting the response to steroid therapy, suggesting room for improvement.

It has long been postulated that autoimmune mechanisms are involved in the pathophysiology of UC $[1,5$, 6], although immune targets have not been identified. Recently, Kuwada et al. reported that autoantibodies against integrin $\alpha v \beta 6$ may be a reliable diagnostic marker for UC [7]. In addition, an association between UC and autoimmune disorders has also been reported [8].

Serum antineutrophil cytoplasmic antibody (ANCA) is a general term for antineutrophil cytoplasmic autoantibodies. Clinically significant ANCAs include cytoplasmic ANCAs (or anti-proteinase 3 (PR3)-ANCA), which target PR3, and perinuclear ANCAs (or myeloperoxidase (MPO)-ANCA), which target the MPO antigen. PR3ANCA is a highly specific biomarker for granulomatosis with polyangiitis [9] and is a highly specific biomarker for eosinophilic granulomatosis with polyangiitis or microscopic polyangiitis [10]. MPO-ANCA is reportedly useful for differentiating UC from Crohn's disease because UC cases in Western countries are often positive for MPOANCA [11-13]. However, in Japan and other regions of Asia, UC cases are commonly positive for PR3-ANCA, but not MPO-ANCA [14, 15]. PR3-ANCA has recently been reported to be useful as a serological marker for evaluating disease severity in UC [15], but the clinical significance of PR3-ANCA in UC has not yet been fully assessed.

UC is diagnosed either histologically or by colonoscopy. Clinical remission has been the primary target of treatment; however, endoscopic remission (when endoscopy shows no mucosal inflammation) has recently been identified as the more appropriate target [16]. An endoscopic evaluation is desirable to evaluate disease activity accurately; however, significant physical and financial burdens make it difficult to perform frequent endoscopic examinations. Fecal hemoglobin and calprotectin have recently been reported to be useful for evaluating disease activity $[17,18]$. However, a serological marker for the assessment of disease activity would be extremely beneficial in clinical practice. Furthermore, a marker of nonresponse to induction therapy could provide physicians with important information.

We conducted a retrospective study to investigate whether PR3-ANCA could be a marker for successful induction therapy with 5-ASA or steroid, and to compare the performance of PR3-ANCA against other markers, including serum CRP and fecal hemoglobin levels.

\section{Methods}

\section{Study design and patients}

We conducted a multicenter retrospective study at the Kagawa Prefectural Central Hospital and Mitoyo General Hospital. We measured PR3-ANCA levels at the time of diagnosis of UC or when treatment was changed to exclude intestinal lesions caused by vasculitis. The inclusion criteria of this study were patients with active UC who underwent colonoscopy and PR3-ANCA measurement within 1 week between April 2016 and March 2020. A total of 173 consecutive patients were included in this study. Key exclusion criteria included being aged below five years; having a complication that could cause PR3-ANCA or MPO-ANCA positivity, such as an autoimmune disease, chronic extraintestinal inflammatory disease [19], or a malignant tumor [20]; already being initiated on drug therapy, including steroids, biologics such as tumor necrosis factor- $\alpha$ inhibitor, tacrolimus, or Janus kinase inhibitor; or patients who declined to participate by opting out. We defined patients under the age of 17 as children. A certified rheumatologist confirmed that no patient had granulomatosis with polyangiitis during UC.

\section{Diagnosis and disease activity of UC}

UC was diagnosed based on the Lennard-Jane criteria [21]. Disease types were classified based on the Montreal Classification [22] as proctitis, left-sided, and extensive. According to colonoscopy results, disease activity was evaluated by assigning the Mayo Endoscopic Subscore (MES) [23] to the sites with the most severely inflamed mucosa. An MES of 0 was defined as remission, and an MES $\geq 1$ was defined as an active-phase disease. The 
pathological activity was evaluated by assigning the Matts grade [24] to the biopsy results of the site with the most severely inflamed mucosa. By agreement, without referring to past medical records, MES was assessed by two endoscopy specialists, and the Matts grade was determined by two pathologists.

Clinical activity was evaluated using the partial Mayo score, which consists of the frequency of defecation, rectal bleeding, and physician rating. A score of $\leq 1$ was defined as clinical remission, and a score of $2-4,5-7$, and $>8$ was defined as mild, moderate, and severe conditions in the active phase, respectively.

\section{Response to the induction therapy}

We examined the response to induction therapy for active-phase colitis in each participant. Those who achieved clinical remission within 90-120 days after starting induction therapy were considered to be responders. Non-response to steroid therapy consisted of steroid resistance and steroid dependence. Steroid resistance is defined as an exhibition of no clinical improvement after treatment with high-dose oral steroids $(40-60 \mathrm{mg} /$ day prednisone or equivalent) within 30 days or no clinical improvement after treatment with high-dose intravenous prednisone within 7-10 days [25]. Steroid dependence is defined as an initial response to treatment with high-dose prednisone, but then relapse during tapering or shortly after drug discontinuation and requiring re-introduction to maintain symptom control [26].

\section{Measurement}

PR3-ANCA and MPO-ANCA levels were measured at an external institution using a kit (STACIA $^{\circledR}$ MEBLux $^{\text {TM }}$ test; MBL) that included a chemiluminescent enzyme immunoassay (CLEIA) on serum that was cryopreserved at $-10{ }^{\circ} \mathrm{C}$ or below. The detection limit values for PR3ANCA and MPO-ANCA were $1.0 \mathrm{U} / \mathrm{mL}$. The cut-off value for PR3-ANCA is $3.5 \mathrm{U} / \mathrm{mL}$ [15]. Serum C-reactive protein (CRP) (cut-off value, $0.14 \mathrm{mg} / \mathrm{dL}$ ) and fecal hemoglobin (cut-off value, $100 \mathrm{ng} / \mathrm{mL}$ ) were selected as activity indicators of the clinical test. Serum CRP levels were measured at the same time as PR3-ANCA, and stool samples for fecal hemoglobin were obtained within two or three days before colonoscopy. Cytomegalovirus (CMV) infection was defined as positive if either CMV immunostaining of biopsy tissue or blood CMV pp65 antigen (C7-HRP) was positive.

\section{Endpoints}

The primary endpoint of this study was to investigate whether PR3-ANCA reflects the success of induction therapy. The secondary endpoint was to reveal the difference between PR3-ANCA and other inflammatory markers, such as serum CRP and fecal hemoglobin levels.

\section{Statistical analysis}

We described the characteristics of patients with the number and percentage for categorical variables and mean with standard deviation or median (range) for continuous variables according to the distribution. We used Fisher's exact test or the Chi-squared test for categorical variables and the Mann-Whitney U test or Student's t-test for continuous variables. We used Dunnett's test for comparisons of PR3-ANCA among MES using MES 3 as a reference, and the same comparisons were performed for CRP and fecal hemoglobin. Spearman's rank correlation was also used for correlations between UC activity and serum PR3-ANCA, serum CRP, and fecal hemoglobin levels. Multivariate logistic regression analysis was used to clarify the factors associated with non-response to steroid therapy. The level of statistical significance was set at a two-sided $P$ value of $<0.05$. EZR (version 3.4.1, Windows) was used for the statistical analyses.

\section{Results}

Of the 173 patients with UC, 159 were analyzed. Of the patients excluded, two were already being treated with steroids, and 12 had either an autoimmune disease, chronic extraintestinal inflammatory disease, or a malignant tumor. Of the 159 patients, 41 (25.8\%) presented with an initial attack of colitis. Patients included 80 men and 79 women with a mean age of 43.5 years ( \pm 17.2 years). The mean age of onset was 36.6 years ( \pm 16.5 years), and the median disease duration was 4.0 years $(1.0-11.0)$. Seven children aged 11, 13,13, 15, 16,16 , and 16 were included in the study. There were 107 cases of extensive colitis (67.3\%), 39 cases of left-sided colitis $(24.5 \%)$, and 13 cases of proctitis (8.2\%) (Table 1 ). Endoscopic images showed classic UC findings, and biopsy results showed no evidence of vasculitis. Thirtyfive patients had an MES of 1, 49 had an MES of 2, and 75 had an MES of 3.

Table 2 shows patients' PR3-ANCA or MPO-ANCA levels grouped by MES. Of the 159 patients, 85 (53.5\%) had serum PR3-ANCA $\geq 3.5 \mathrm{U} / \mathrm{mL}$, indicating the PR3-ANCA-positive group, and 74 (46.5\%) had serum PR3-ANCA $<3.5 \mathrm{U} / \mathrm{mL}$, indicating the negative group. The PR3-ANCA-positive group had significantly higher MES and pathological activity $(P=0.032$ and 0.013 , respectively) than the negative group (Table 3 ). In seven child patients, three were PR3-ANCA-positive and none were MPO-ANCA-positive. 
Table 1 Characteristics of patients

\begin{tabular}{|c|c|}
\hline & $n=159$ \\
\hline Age, mean $\pm S D$ (years) & $43.5 \pm 17.2$ \\
\hline Sex, male/female & $80(50.3 \%) / 79(49.7 \%)$ \\
\hline $\mathrm{BMI}$, mean $\pm \mathrm{SD}\left(\mathrm{kg} / \mathrm{m}^{2}\right)$ & $22.4 \pm 3.5$ \\
\hline History of smoking & $52(32.7 \%)$ \\
\hline Current alcohol consumption & $41(25.8 \%)$ \\
\hline Age at onset, mean \pm SD (years) & $36.6 \pm 16.5$ \\
\hline Disease duration, median (range), years & $4.0(1.0-11.0)$ \\
\hline Initial attack & $41(25.8 \%)$ \\
\hline \multicolumn{2}{|l|}{ Type } \\
\hline Extensive & $107(67.3 \%)$ \\
\hline Left-sided & $39(24.5 \%)$ \\
\hline Proctitis & $13(8.2 \%)$ \\
\hline Frequency of defecation per day, median (range) & $5(3-8)$ \\
\hline Bloody stool & $95(59.7 \%)$ \\
\hline CRP, median (range), mg/dL & $0.19(0.05-1.31)$ \\
\hline Alb, median (range), g/dL & $4.1(3.7-4.4)$ \\
\hline Plt, median (range), $\times 10^{4} / \mu \mathrm{L}$ & $30.0(24.4-38.4)$ \\
\hline Fecal hemoglobin, median (range), ng/mL & $1559(144-5284)$ \\
\hline CMV infection & $9(5.7 \%)$ \\
\hline MES, median (range) & $2(1-3)$ \\
\hline Matts grade, median (range) & $4(3-5)$ \\
\hline
\end{tabular}

SD, standard deviation; BMI, body mass index; CRP, C-reactive protein; Alb, albumin; PIt, platelet; CMV, cytomegalovirus; MES, Mayo Endoscopic Subscore

Table 2 Patients' PR3-ANCA and MPO-ANCA levels by MES group

\begin{tabular}{lccc}
\hline MES & $\mathbf{1}(\mathbf{n}=\mathbf{3 5})$ & $\mathbf{2}(\mathbf{n}=\mathbf{4 9})$ & $\mathbf{3}(\mathbf{n}=\mathbf{7 5})$ \\
\hline PR3-ANCA $(\geq 3.5)$ & 14 & 24 & 47 \\
PR3-ANCA $(<3.5)$ & 21 & 25 & 28 \\
MPO-ANCA $(\geq 1.0)$ & 2 & 4 & 4 \\
MPO-ANCA $(<1.0)$ & 33 & 45 & 71 \\
\hline
\end{tabular}

MES, Mayo Endoscopic Subscore; PR3-ANCA, anti-proteinase 3 antineutrophil cytoplasmic antibody; MPO-ANCA, myeloperoxidase antineutrophil cytoplasmic antibody

\section{Relationship between MES versus CRP and fecal hemoglobin}

The median CRP titers at MES 1, 2, and 3 were 0.05 (0.03-0.21), $0.11(0.04-0.4)$, and $0.83(0.15-5.9)$, respectively. There was a statistically significant difference in CRP titers between MES 3 and 2, or MES 3 and 1 (both $P<0.001)$. A positive correlation was observed between the MES and CRP levels $(r=0.444 ; P<0.001)$ (Fig. 1a).

To assess the fecal hemoglobin levels, 115 patients were analyzed. Of 159 participants, 44 (27.7\%) could not provide stool samples because of sampling difficulty in the active phase of the disease. The median fecal hemoglobin titers at MES 1, 2, and 3 were 0 (0-417), 853 (270-4290), and 4031 (1173-8090), respectively. There was a statistically significant difference in fecal hemoglobin titer between MES 3 and $1(P<0.001)$, but there was no significant difference between MES 3 and $2(P=0.052)$. A positive correlation was observed between MES and the fecal hemoglobin level $(r=0.469 ; P<0.001)$ (Fig. 1b).

\section{Relationship between MES or Matts grade versus PR3-ANCA}

The median PR3-ANCA titers at MES 1, 2, and 3 were 1.1 (0-7.6), 3.4 (1.0-10.1), and 6.1 (2.1-18.1), respectively. There was a statistically significant difference in PR3-ANCA titers between MES 3 and $1(P=0.002)$ and between MES 3 and $2(P=0.035)$. A positive correlation was observed between MES and serum PR3-ANCA levels $(r=0.228 ; 95 \%$ confidence interval $[\mathrm{CI}], 0.075-0.371$; $P=0.004)$ (Fig. 1c).

Of the 159 patients, eight had a Matts grade of 1 (5.0\%), 20 had a Matts grade of $2(12.6 \%), 43$ had a Matts grade of $3(27.1 \%), 36$ had a Matts grade of $4(22.6 \%)$, and 52 had a Matts grade of $5(32.7 \%)$. Titers of PR3-ANCA at Matts grade $1,2,3,4$, and 5 were $0(0-3.2), 1.4(0-10.5), 4.1$ (0-22.5), $3.3(0.8-8.4)$, and $6.3(2.4-16.8)$, respectively. A positive correlation was observed between the Matts grade and serum PR3-ANCA levels $(r=0.234 ; P=0.003)$.

\section{Relationship between PR3-ANCA versus CRP and fecal hemoglobin}

No positive correlation was observed between PR3ANCA and CRP levels $(\mathrm{r}=0.152 ; P=0.056)$. CRP positivity in 85 PR3-ANCA-positive patients was not different from that of 74 PR3-ANCA-negative patients (53\% vs. $38 \%, P=0.199)$. No positive correlation was observed between PR3-ANCA and fecal hemoglobin $(\mathrm{r}=0.17$; $P=0.071$ ). Fecal hemoglobin positivity in 85 PR3-ANCApositive patients was the same as that in 74 PR3-ANCAnegative patients $(57.6 \%$ vs. $54.1 \%, P=0.749)$.

\section{PR3-ANCA as a predictor of non-response to the induction} therapy

The induction therapy response for active-phase colitis in each participant was assessed 98 days after starting the treatment. The assessment was performed between day 91 and 112. Therapy with 5-ASA was introduced in 69 patients with a median partial Mayo score of 5 (3-6). Steroid therapy was introduced in 56 patients with a median partial Mayo score of 7 (5-9).

Figure 2 shows PR3-ANCA positivity in responders and non-responders to treatment with 5-ASA or steroid therapy. The proportion of PR3-ANCA positivity was not significantly different with respect to the response to 5-ASA therapy (50\% of responders vs. $52 \%$ of non-responders, 
Table 3 Comparison of the PR3-ANCA-positive and negative groups in the active phase

\begin{tabular}{|c|c|c|c|}
\hline & $\begin{array}{l}\text { PR3-ANCA positive } \\
(\mathrm{n}=85)\end{array}$ & $\begin{array}{l}\text { PR3-ANCA negative } \\
(\mathrm{n}=74)\end{array}$ & $P$ value \\
\hline Age, mean $\pm S D$ (years) & $40.8 \pm 17.2$ & $46.6 \pm 16.7$ & 0.034 \\
\hline Sex, male/female & $45(52.9 \%) / 40(47.1 \%)$ & $39(52.7 \%) / 35(47.3 \%)$ & 1 \\
\hline $\mathrm{BMI}$, mean $\pm \mathrm{SD}\left(\mathrm{kg} / \mathrm{m}^{2}\right)$ & $22.4 \pm 3.2$ & $22.3 \pm 3.7$ & 0.89 \\
\hline History of smoking & $24(28.2 \%)$ & $28(37.8 \%)$ & 0.24 \\
\hline Current alcohol consumption & $22(25.9 \%)$ & $19(25.7 \%)$ & 1 \\
\hline Age at onset, mean \pm SD (years) & $35.3 \pm 16.9$ & $38.2 \pm 16.0$ & 0.27 \\
\hline Disease duration, median (range), years & $3.0(0-8)$ & $6.0(1-13)$ & 0.083 \\
\hline Type & & & 0.28 \\
\hline Extensive & $62(72.9 \%)$ & $45(60.8 \%)$ & \\
\hline Left-sided & $17(20.0 \%)$ & $22(29.7 \%)$ & \\
\hline Proctitis & $6(7.1 \%)$ & $7(9.5 \%)$ & \\
\hline Frequency of defecation per day, median (range) & $5(3-8)$ & $4(2-7)$ & 0.11 \\
\hline Bloody stool & $54(63.5 \%)$ & $41(55.4 \%)$ & 0.33 \\
\hline CRP, median (range), mg/dL & $0.32(0.06-1.39)$ & $0.15(0.04-0.87)$ & 0.15 \\
\hline Alb, median (range), g/dL & $4.0(3.2-4.4)$ & $4.2(3.8-4.4)$ & 0.089 \\
\hline Plt, median (range) $\times 10^{4} / \mu \mathrm{L}$ & $31.2(25.4-40.2)$ & $29.9(22.6-35.5)$ & 0.20 \\
\hline Fecal hemoglobin, median (range), ng/mL & $1703(264-6054)$ & $1158(137-4675)$ & 0.278 \\
\hline CMV infection & $5(5.9 \%)$ & $4(5.4 \%)$ & 1 \\
\hline MES, median (range) & $3(2-3)$ & $2(1-3)$ & 0.032 \\
\hline Matts grade, median (range) & $4(3-5)$ & $3(3-4)$ & 0.013 \\
\hline
\end{tabular}

SD, standard deviation; BMI, body mass index; CRP, C-reactive protein; Alb, albumin; Plt, platelet; CMV, cytomegalovirus; MES, Mayo Endoscopic Subscore
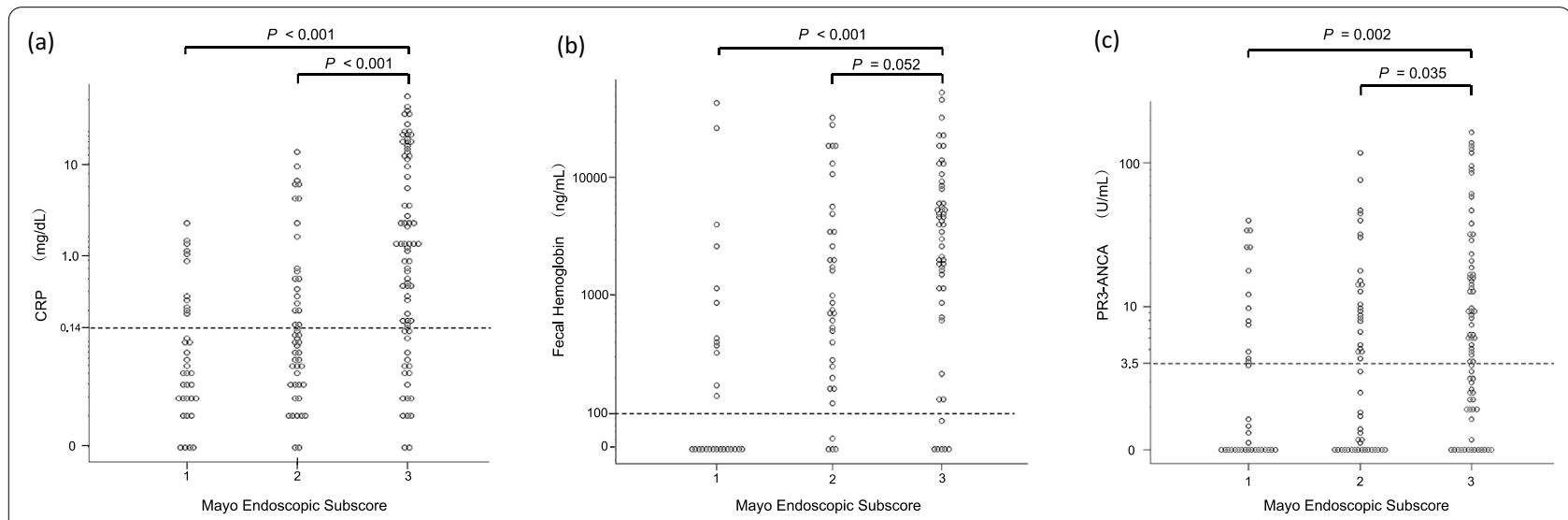

Fig. 1 Correlation between MES versus serum CRP levels (a), fecal hemoglobin levels (b), and serum PR3-ANCA levels (c). MES, Mayo Endoscopic Subscore; CRP, C-reactive protein; PR3-ANCA, anti-proteinase 3 antineutrophil cytoplasmic antibody

$P=1)$. PR3-ANCA positivity in non-responders to steroid therapy was significantly higher than that in responders $(71.9 \%$ vs. $41.7 \%, P=0.030)$. PR3-ANCA positivity in non-responders to steroid therapy remained high, even after the exclusion of four child patients $(72.4 \%$ vs. $43.5 \%$, $P=0.048)$.

CRP positivity of non-responders to steroid therapy was not different from that of responders $(78.1 \%$ vs.
$58.3 \%, P=0.146)$. Fecal hemoglobin positivity of nonresponders to steroid therapy was not different from that of responders $(65.6 \%$ vs. $54.2 \%, P=0.42)$.

Table 4 shows the comparison between responders and non-responders to 5-ASA therapy. The median partial Mayo scores of responders and non-responders were 4 (3-6) and 5 (4-6), respectively, without a significant difference $(P=0.55)$. The median PR3-ANCA titers in 


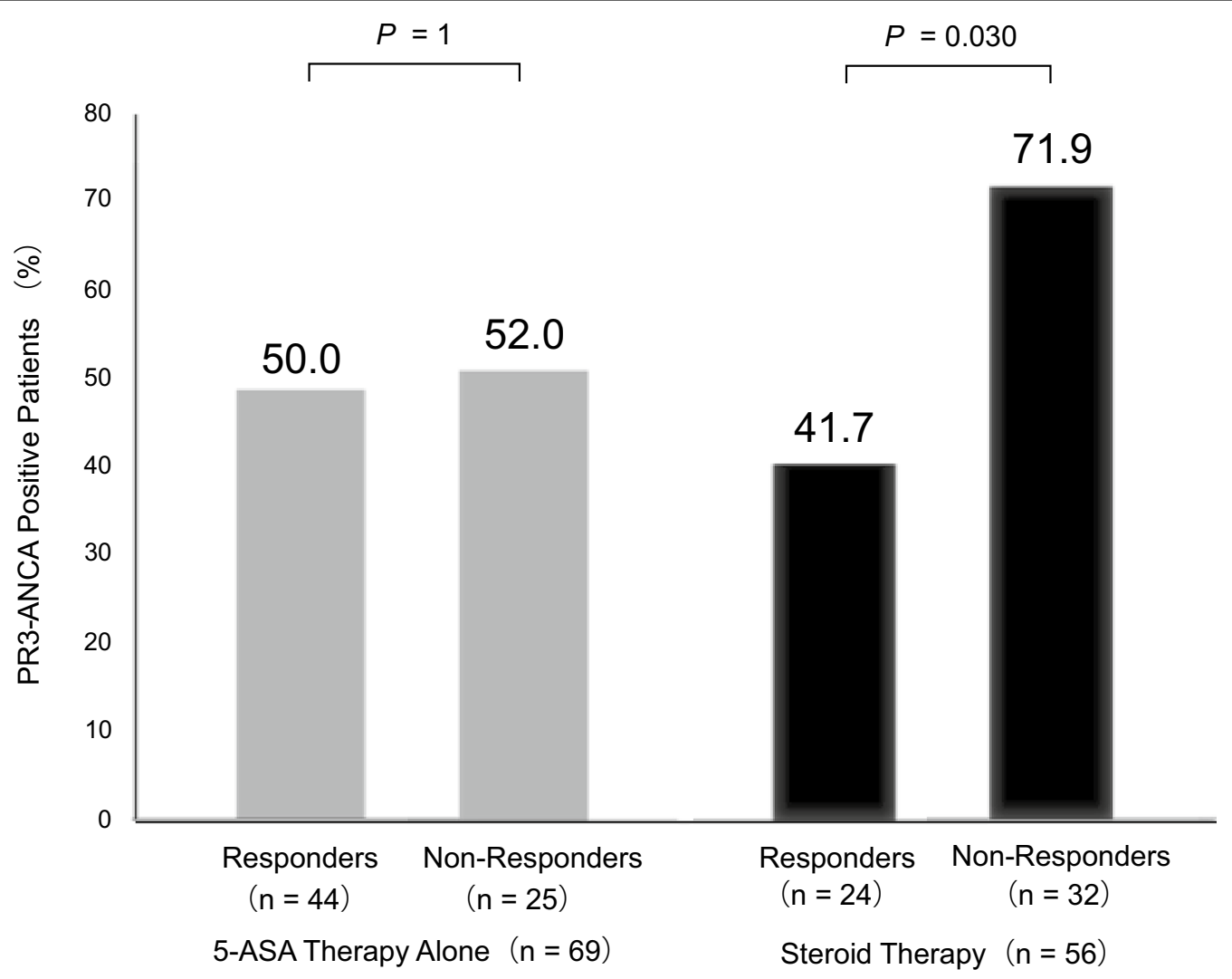

Fig. 2 PR3-ANCA positivity in responders and non-responders to induction therapy. 5-ASA, 5-aminosalicylic acid; PR3-ANCA, anti-proteinase 3 antineutrophil cytoplasmic antibody

responders and non-responders to 5-ASA therapy were 2.5 (1.4-14.8) and $3.9(1.7-14.2)$, respectively, without a statistically significant difference $(P=0.20)$. Of the nonresponders to 5-ASA therapy, the proportions of those who were PR3-ANCA-positive were $50.0 \%$ (2/4) who were MES 1, 28.6\% (2/7) who were MES 2, and $64.3 \%$ (9/14) who were MES 3.

Table 5 shows the comparison between responders and non-responders to steroid therapy. The median partial Mayo scores of 24 responders and 32 non-responders were 6 (5-7) and 7 (5-9), respectively, without a significant difference $(P=0.45)$. The median PR3-ANCA titers in responders and non-responders to steroid therapy were $1.9(1.2-7.5)$ and $8.9(1.9-25.5)$, respectively, with a statistically significant difference $(P=0.041)$. Of the nonresponders to steroid therapy, the percentage of those who were PR3-ANCA positive was $0 \%(0 / 1)$ for MES 1 , 83.3\% (5/6) for MES 2, and 76.0\% (18/25) for MES 3.

Among 32 non-responders to steroid therapy, the median PR3-ANCA titers in 14 steroid-resistant and 18 steroid-dependent patients were 11.0 (6.7-51.3) and $6.1(1.2-12.0)$, respectively. PR3-ANCA titers in steroid-resistant patients were higher than those in steroid-dependent patients, but the difference was not statistically significant $(P=0.105)$.

\section{Performance of PR3-ANCA for predicting a non-response to steroid therapy}

Univariate analysis revealed significant differences between responders and non-responders to steroid therapy in the frequency of defecation (median [range]: 5 [3-8] vs. 8 [5-12], $P=0.017)$, bloody stool $(45.8 \%$ $[\mathrm{n}=11]$ vs. $84.4 \%[\mathrm{n}=27], P=0.004)$, albumin titers (4.1 [3.6-4.4] vs. 3.4 [2.7-4.1], $P=0.006)$, Matts grade (4 [3-5] vs. $5[4,5], P=0.015)$, PR3-ANCA positivity (41.7\% $[\mathrm{n}=10]$ vs. $71.9 \%[\mathrm{n}=23], P=0.030)$, and PR3ANCA titers $(1.9[1.2-7.5]$ vs. 8.9 [1.9-25.5], $P=0.041)$ (Table 5).

Multivariate analysis using logistic regression analysis showed that PR3-ANCA positivity was significantly associated with a non-response to steroid therapy (odds ratio $5.19,95 \% \mathrm{CI}: 1.54-17.5 ; P=0.008)$. No other factors, including CRP, fecal hemoglobin, disease duration, and partial Mayo score, were significantly associated with a non-response to steroid therapy (Table 6). 
Table 4 Comparison of responders and non-responders to 5-ASA therapy

\begin{tabular}{|c|c|c|c|}
\hline & $\begin{array}{l}\text { 5-ASA responders } \\
(n=44)\end{array}$ & $\begin{array}{l}\text { 5-ASA non-responders } \\
(\mathrm{n}=25)\end{array}$ & $P$ value \\
\hline Age, mean $\pm S D$ (years) & $42.8 \pm 16.8$ & $49.0 \pm 17.6$ & 0.16 \\
\hline Sex, M/F & $24(54.5 \%) / 20(45.5 \%)$ & $12(48.0 \%) / 13(52.0 \%)$ & 0.625 \\
\hline $\mathrm{BMI}$, mean $\pm \mathrm{SD}\left(\mathrm{kg} / \mathrm{m}^{2}\right)$ & $21.7 \pm 2.5$ & $22.2 \pm 3.1$ & 0.49 \\
\hline History of smoking & $17(38.6 \%)$ & $5(20.0 \%)$ & 0.18 \\
\hline Current alcohol consumption & $13(29.5 \%)$ & $8(32.0 \%)$ & 1 \\
\hline Age at onset, mean \pm SD (years) & $35.1 \pm 14.8$ & $42.8 \pm 17.2$ & 0.064 \\
\hline Disease duration, median (range), years & $3.0(1-12)$ & $3.0(1-8)$ & 0.55 \\
\hline Type & & & 1 \\
\hline Extensive & $24(54.5 \%)$ & $15(60.0 \%)$ & \\
\hline Left-sided & $15(34.1 \%)$ & $8(32.0 \%)$ & \\
\hline Proctitis & $5(11.4 \%)$ & $2(8.0 \%)$ & \\
\hline Frequency of defecation per day, median (range) & $4(1-6)$ & $5(4-8)$ & 0.046 \\
\hline Bloody stool & $20(45.5 \%)$ & $19(76.0 \%)$ & 0.022 \\
\hline CRP, median (range), mg/dL & $0.08(0.04-0.33)$ & $0.57(0.09-2.8)$ & 0.011 \\
\hline Alb, median (range), g/dL & $4.3(4.1-4.5)$ & $3.9(3.2-4.1)$ & $<0.001$ \\
\hline Plt, median (range) $\times 10^{4} / \mu \mathrm{L}$ & $29.5(22.5-33.4)$ & $31.4(26.9-36.8)$ & 0.19 \\
\hline Fecal hemoglobin, median (range), ng/mL & $710(122-3938)$ & $5608(676-14,270)$ & 0.012 \\
\hline CMV infection & $0(0 \%)$ & $1(25.0 \%)$ & 0.36 \\
\hline MES, median (range) & $2(1-3)$ & $3(2-3)$ & 0.005 \\
\hline Matts grade, median (range) & $3(2-4)$ & $4(4-5)$ & $<0.001$ \\
\hline Partial Mayo score & $4(3-6)$ & $5(4-6)$ & 0.55 \\
\hline PR3-ANCA positive & $21(47.7 \%)$ & $13(52.0 \%)$ & 0.81 \\
\hline PR3-ANCA, median (range), $\mathrm{U} / \mathrm{mL}$ & $2.5(1.4-14.8)$ & $3.9(1.7-14.2)$ & 0.20 \\
\hline
\end{tabular}

BMI, body mass index; CRP, C-reactive protein; Alb, albumin; PIt, platelet; PR3-ANCA, anti-proteinase 3 anti-neutrophil cytoplasmic antibody; MPO-ANCA, myeloperoxidase anti-neutrophil cytoplasmic antibody; CMV, cytomegalovirus; MES, Mayo endoscopic subscore

\section{Change of PR3-ANCA in responders and non-responders to steroid therapy}

Of the 24 responders to steroid therapy, 10 patients (41.7\%) were initially positive for PR3-ANCA and were successfully treated for clinical remission with steroids. The median titer of PR3-ANCA-positive responders to steroids was $14.1(8.2-19.0)$ during the active phase, and became significantly lower at $1.5(1.0-4.2)$ in clinical remission $(P=0.002)$. In $70.0 \%(7 / 10)$ of PR3-ANCApositive responders, PR3-ANCA became negative in clinical remission.

Additional therapy was administered to 32 nonresponders to steroid therapy. Among these patients, 23 (71.9\%) were PR3-ANCA positive. Nineteen patients (82.6\%) of these 23 PR3-ANCA-positive non-responders were successfully treated for clinical remission. Biologics, including tumor necrosis factor- $\alpha$ inhibitor, reinduction of high-dose steroids, tacrolimus, and Janus kinase inhibitor with leukocytapheresis (LCAP), achieved clinical remission in 11 of 13 patients (84.6\%), in five of seven patients $(71.4 \%)$, in two of two patients $(100 \%)$, and in one of one patient (100\%), respectively.
The median titer of PR3-ANCA in PR3-ANCA-positive non-responders was $23.0(7.6-69.2)$ in the active phase and $6.7(2.0-32.1)$ in clinical remission. The reduction in PR3-ANCA levels was significant when comparing the active phase and clinical remission $(P<0.001)$. However, PR3-ANCA became negative in only $36.8 \%(7 / 19)$ of PR3-ANCA-positive non-responders during clinical remission.

\section{Changes in PR3-ANCA, CRP, or fecal hemoglobin levels in patients who underwent follow-up colonoscopy in clinical remission}

Fifty-eight patients (active phase PR3-ANCA-positive cases) were observed for 7.6 months (5.3-14.7) and underwent endoscopic examination and measurements of PR3-ANCA, CRP, and fecal hemoglobin. Clinical remission was achieved in 37 of the 58 cases $(63.8 \%)$, and the relationships between MES, which was assessed in clinical remission versus serum PR3-ANCA, serum CRP, and fecal hemoglobin levels, were analyzed in these 37 patients (Table 7). Twenty-seven patients had an MES $\geq 1$, and only 10 patients had an MES of 0 . 
Table 5 Comparison of responders and non-responders to steroid therapy

\begin{tabular}{|c|c|c|c|}
\hline & $\begin{array}{l}\text { Steroid responders } \\
(n=24)\end{array}$ & $\begin{array}{l}\text { Steroid non-responders } \\
(n=32)\end{array}$ & $P$ value \\
\hline & & $\begin{array}{l}14 \text { steroid-resistant } \\
18 \text { steroid-dependent }\end{array}$ & \\
\hline Age, mean $\pm S D$ (years) & $46.1 \pm 17.8$ & $43.5 \pm 19.8$ & 0.606 \\
\hline Sex, M/F & $15(62.5 \%) / 9(37.5 \%)$ & $16(50.0 \%) / 16(50.0 \%)$ & 0.421 \\
\hline $\mathrm{BMI}$, mean $\pm \mathrm{SD}\left(\mathrm{kg} / \mathrm{m}^{2}\right)$ & $23.3 \pm 4.6$ & $23.0 \pm 3.9$ & 0.83 \\
\hline History of smoking & $9(37.5 \%)$ & $11(34.4 \%)$ & 1 \\
\hline Current alcohol consumption & $6(25.0 \%)$ & $8(25.0 \%)$ & 1 \\
\hline Age at onset, mean \pm SD (years) & $38.9 \pm 17.7$ & $37.8 \pm 19.9$ & 0.82 \\
\hline Disease duration, median (range), years & $6.0(0-9)$ & $3.5(1-9.5)$ & 0.71 \\
\hline Type & & & 1 \\
\hline Extensive & $20(83.3 \%)$ & $27(84.4 \%)$ & \\
\hline Left-sided & $4(16.7 \%)$ & $5(15.6 \%)$ & \\
\hline Proctitis & $0(0 \%)$ & $0(0 \%)$ & \\
\hline Frequency of defecation per day, median (range) & $5(3-8)$ & $8(5-12)$ & 0.017 \\
\hline Bloody stool & $11(45.8 \%)$ & $27(84.4 \%)$ & 0.004 \\
\hline CRP, median (range), mg/dL & $0.29(0.05-2.1)$ & $1.18(0.35-6.0)$ & 0.074 \\
\hline Alb, median (range), g/dL & $4.1(3.6-4.4)$ & $3.4(2.7-4.1)$ & 0.006 \\
\hline Plt, median (range) $\times 10^{4} / \mu \mathrm{L}$ & $29.9(21.8-37.8)$ & $39.0(28.3-45.6)$ & 0.066 \\
\hline Fecal hemoglobin, median (range), ng/mL & $999(50-5664)$ & $3630(1329-9417)$ & 0.074 \\
\hline CMV infection & $2(8.3 \%)$ & $3(9.4 \%)$ & 1 \\
\hline MES, median (range) & $3(2-3)$ & $3(3-3)$ & 0.081 \\
\hline Matts grade, median (range) & $4(3-5)$ & $5(4-5)$ & 0.015 \\
\hline Partial Mayo score & $6(5-7)$ & $7(5-9)$ & 0.45 \\
\hline PR3-ANCA positive & $10(41.7 \%)$ & $23(71.9 \%)$ & 0.030 \\
\hline PR3-ANCA, median (range), $\mathrm{U} / \mathrm{mL}$ & $1.9(1.2-7.5)$ & $8.9(1.9-25.5)$ & 0.041 \\
\hline
\end{tabular}

BMI, body mass index; CRP, C-reactive protein; Alb, albumin; PIt, platelet; PR3-ANCA, anti-proteinase 3 anti-neutrophil cytoplasmic antibody; MPO-ANCA, myeloperoxidase anti-neutrophil cytoplasmic antibody; CMV, cytomegalovirus; MES, Mayo Endoscopic Subscore

Table 6 The association between a non-response to steroid therapy and explanatory variables

\begin{tabular}{llll}
\hline & Odds ratio & $\begin{array}{l}\text { 95\% Confidence } \\
\text { interval }\end{array}$ & $\boldsymbol{P}$ value \\
\hline Disease duration & 1.01 & $0.92-1.10$ & 0.871 \\
CRP & 0.97 & $0.81-1.15$ & 0.693 \\
Fecal hemoglobin & 1.00 & $1.00-1.00$ & 0.804 \\
Partial Mayo score & 1.35 & $0.97-1.87$ & 0.077 \\
PR3-ANCA positive & 5.19 & $1.54-17.50$ & 0.008 \\
\hline
\end{tabular}

CRP, C-reactive protein; PR3-ANCA, anti-proteinase 3 anti-neutrophil cytoplasmic antibody

For cases with MES 0, seven were treated with steroids, two were treated with biologics, and one was treated with tacrolimus as induction therapy. For MES $\geq 1$ patients, 12 were treated with 5-ASA, 10 were treated with steroids, two were treated with biologics, two were treated with tacrolimus, and one was treated with 5-ASA plus LCAP as induction therapy. During the observation period,
Table 7 PR3-ANCA, CRP, and fecal hemoglobin levels by MES group in clinical remission cases

\begin{tabular}{lclll}
\hline MES & $\mathbf{0}(\mathbf{n}=\mathbf{1 0 )})$ & $\mathbf{1}(\mathbf{n}=\mathbf{9})$ & $\mathbf{2}(\mathbf{n}=\mathbf{1 6})$ & $\mathbf{3}(\mathbf{n}=\mathbf{2})$ \\
\hline $\begin{array}{l}\text { PR3-ANCA positive, } \\
(n=20)\end{array}$ & 2 & 5 & 11 & 2 \\
$\begin{array}{l}\text { PR3-ANCA negative, } \\
(n=17)\end{array}$ & 8 & 4 & 5 & 0 \\
CRP positive, $(n=10)$ & 0 & 3 & 7 & 0 \\
CRP negative, $(n=27)$ & 10 & 6 & 9 & 2 \\
FH positive, $(n=15)$ & 1 & 2 & 11 & 1 \\
FH negative, $(n=18)$ & 7 & 6 & 5 & 0
\end{tabular}

MES, Mayo Endoscopic Subscore; PR3-ANCA, anti-proteinase 3 antineutrophil cytoplasmic antibody; $\mathrm{CRP}, \mathrm{C}$-reactive protein; $\mathrm{FH}$, fecal hemoglobin

maintenance therapy was almost the same in MES 0 and MES $\geq 1$ patients.

The reductions in CRP levels in cases with MES 0 and $M E S \geq 1$ were not significant when comparing the active phase and clinical remission $(P=0.148$ and $P=0.098$, respectively) (Fig. 3a). The reductions in fecal 

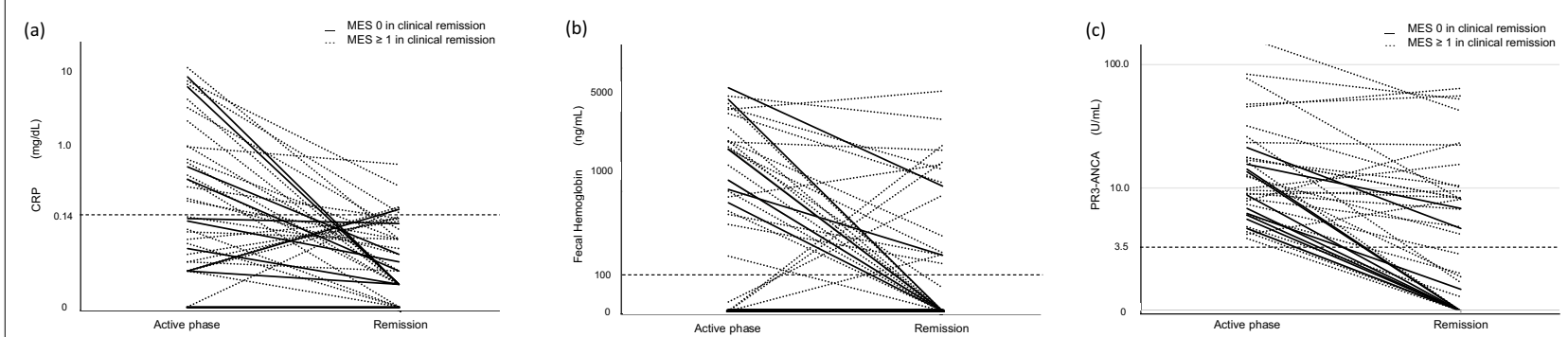

Fig. 3 Changes in CRP titers (a), fecal hemoglobin titers (b), and PR3-ANCA titers (c) in cases that reached clinical remission. Significant reductions in the levels were observed in cases with MES 0 and MES $\geq 1$ for fecal hemoglobin and PR3-ANCA when comparing the active phase and clinical remission ( $P=0.036$ and $P=0.015, P<0.001$ and $P=0.043$, respectively). CRP, C-reactive protein; PR3-ANCA, anti-proteinase 3 antineutrophil cytoplasmic antibody; MES, Mayo Endoscopic Subscore

hemoglobin levels in cases with MES 0 and MES $\geq 1$ were significant when comparing the active phase with clinical remission $(P=0.036$ and $P=0.015$, respectively) (Fig. 3b). The reductions in PR3-ANCA levels in cases with MES 0 and MES $\geq 1$ were significant when comparing the active phase and clinical remission $(P<0.001$ and $P=0.043$, respectively) (Fig. 3c). For cases with MES 0 , the median titer of PR3-ANCA was 7.8 (6.1-14.0) in the clinical active phase and $0(0-1.4)$ in clinical remission. For $\mathrm{MES} \geq 1$ patients, the median titer of PR3-ANCA was $12.3(7.6-24.7)$ in the clinical active phase and 8.1 (2.0-12.9) in clinical remission.

In clinical remission, the fecal hemoglobin in 33 patients became negative in 18 patients and remained positive in 15 patients. The ratio of MES 0 in 18 patients whose fecal hemoglobin became negative was higher than that in 15 patients whose fecal hemoglobin remained positive with a statistically significant difference $(38.9 \%$ vs. $6.7 \%, P=0.046)$.

\section{Significance of PR3-ANCA as a target for treatment}

Although clinical remission was achieved in 37 cases, PR3-ANCA was negative in 17 patients and remained positive in 20 patients. The ratio of MES 0 in the 17 patients whose PR3-ANCA became negative was higher than that in the 20 patients whose PR3-ANCA remained positive with a statistically significant difference $(47.1 \%$ vs $10.0 \%, P=0.023)$. Concerning detectability of the mucosal healing equivalent to MES 0, PR3-ANCA had a sensitivity of $66.7 \%$, a specificity of $80.0 \%$, a positive predictive value of $90.0 \%$, a negative predictive value of $47.1 \%$, and a proper diagnosis rate of $70.3 \%$.

\section{Discussion}

In this study, UC patients who were non-responders to steroid therapy had higher positive rates and values for PR3-ANCA than responders. In contrast, the positivity of CRP and fecal hemoglobin in non-responders to steroid therapy was not different from that of responders. Multivariate analysis using logistic regression analysis demonstrated that PR3-ANCA positivity was associated with non-response to steroid therapy (odds ratio 5.19). Concerning the inflammatory markers, PR3-ANCA, CRP, and fecal hemoglobin were correlated with disease activity based on MES in the active phase. However, a positive correlation was not observed between PR3-ANCA and CRP levels and fecal hemoglobin. PR3-ANCA and fecal hemoglobin levels reflected endoscopic activity in clinical remission.

CRP is primarily dependent on liver production induced by circulating interleukin 6 , and only modest to absent CRP responses are observed in UC, despite active inflammation in the colon [27]. In this study, the usefulness of CRP as a marker of disease activity was limited. Fecal calprotectin is commonly used to evaluate disease activity in Western countries. However, we used fecal hemoglobin as a marker in this study because fecal calprotectin was not available in Japan for clinical use. Fecal hemoglobin is a useful marker of disease activity in UC, which directly reflects the level of mucosal damage of the colon [17] and reflects the inflammation of the mucosa in this study.

Several serologic markers for disease activity of UC, such as trefoil factor 3 [28] and leucine-rich alpha-2 glycoprotein (LRG) [29], have been reported. LRG has recently become available in Japan for clinical use, but is affected by several inflammatory factors outside the intestine [30]. Further study is needed to investigate the significance of LRG in the management of patients with UC.

The positivity rate for PR3-ANCA in UC has been reported to be $39.2 \%$ among Japanese individuals by Takedatsu et al. [15]. In addition, this value is reportedly 8.6\% among Chinese individuals, $12.1 \%$ among Swedish individuals [31], and $14.1 \%$ among Spanish individuals [32]. In the present study considering Japanese 
individuals, the PR3-ANCA positivity rate was 53.5\%, which is close to that reported by Takedatsu et al., and higher than that reported in other countries.

Measurements of PR3-ANCA have been known to yield different results depending on the method used. Methods of measuring ANCA can be broadly classified as indirect immunofluorescence (IIF) and enzyme immunoassays (EIA). IIF is reportedly unreliable because of changes in antigenicity or admixture of other antigens [33]. EIA methods include enzyme-linked immunosorbent assays (ELISAs) and CLEIAs. Regarding EIA, CLEIA is reportedly more sensitive than ELISA for the examination of the same samples [34]. Although PR3-ANCA was measured using the same CLEIA method in the present study and by Takedatsu et al. [15], other studies have not described the measurement methods in detail. The differences in the positivity rates of previous studies may be the result of differences in the measurement methods used.

Factors that affect the PR3-ANCA positivity rate in UC include patient background and UC activity. Important patient background characteristics include age and concomitant disease. A higher positive rate of PR3-ANCA, such as $57.6 \%$ [35] compared to that of previous studies on adult patients, and the usefulness for differential diagnosis of Crohn's disease [36] have been reported in children with UC. Further studies are necessary to investigate whether PR3-ANCA predicts a non-response to steroid therapy in children. However, the small number of children included in this study had little effect on the results.

A relationship between PR3-ANCA and other diseases including chronic respiratory disease and malignant tumors has also been reported [19, 20]. Previous investigations of the relationship between UC and PR3ANCA have not considered factors other than UC that could lead to PR3-ANCA-positivity. The present study excluded cases involving complications that could affect PR3-ANCA.

The relationship between UC activity and PR3-ANCA remains controversial. PR3-ANCA-positive UC has been reported to involve a few refractory cases as well as those severe enough to require surgery or intervention with immunoregulators or biologics [34]. However, there have been reports of refractory instances and those that involve primary sclerosing cholangitis as a complication [37]. Furthermore, Takedatsu et al. [15] recently reported that PR3-ANCA is related to UC activity.

Although the underlying cause of UC remains unknown, large quantities of neutrophils are observed at the inflamed sites of the intestinal mucosa. No prior study has investigated how PR3-ANCA affects inflammation of the mucosa caused by UC. Hyperactivity of neutrophils due to the formation and release of neutrophil extracellular traps (NETs) has been discussed as it pertains to the pathology of PR3-ANCA-associated vasculitis [10, 38, 39]. NETs are a network-like structure inside neutrophils with a mixture of neutrophil intranuclear DNA, neutrophil cytoplasmic MPO and PR3, neutrophil elastase, bactericidal/permeability-increasing protein, lactoferrin, and other antimicrobial proteins. In response to infection stimuli, NETs are released outside the cell, trapping and killing pathogens while minimizing host cell death [40]. However, NETs directly injure the vascular endothelial cells. Therefore, they are strictly controlled by being broken down, mainly by deoxyribonuclease I [41]. PR3specific mouse monoclonal antibodies have been shown to assist in the formation of NETs [39]. Overproduction of NETs has been inferred to lower immune tolerance to MPO and PR3, consequently causing ANCA overproduction, which induces neutrophil hyperactivity and promotes inflammation [39, 42-44].

Of the patients in the current study with mild colitis treated with 5-ASA as induction therapy, PR3-ANCA positivity in responders and non-responders was no different at approximately $50 \%$ in the two groups. PR3ANCA is produced regardless of the degree of inflammation in UC. However, in moderate-to-severe colitis patients treated with steroids as induction therapy, PR3ANCA positivity in non-responders was significantly higher than that in responders. Therefore, when inflammation becomes advanced, PR3-ANCA may be more strongly involved in aggravating the inflammatory process and diminishing the therapeutic effect of the drugs; however, the underlying mechanism remains unclear.

ANCA-associated vasculitis is treated with a combination therapy comprising of steroids and immunoregulators [45, 46]. With PR3-ANCA-positive UC, PR3-ANCA may intensify inflammation of the mucosa caused by $\mathrm{UC}$, thus, rendering steroid monotherapy insufficient to treat moderate-to-severe UC. If this is the case, then PR3-ANCA could provide treating physicians with useful information when considering treatment options other than steroid monotherapy. In addition, inhibiting PR3ANCA may be useful as a goal for induction therapy.

This study has several limitations. First, this was a retrospective study with a short follow-up period and a relatively small sample size. Ten responders and 23 nonresponders to steroid therapy were PR3-ANCA-positive, and this small sample size may have been insufficient for multivariate analysis. Second, the observational study could not exclude the effects of other possible confounding factors on non-response to steroid therapy. Third, we made many comparisons within a limited sample size. Although we conducted these analyses based on the predetermined hypothesis, such tests should be interpreted 
cautiously. Fourth, a single method was used to measure serum PR3-ANCA levels. Other measurement methods must be used to investigate whether the results are similar. Fifth, because all patients included in this study were Japanese, it is unclear whether our findings can be applied to other populations. To overcome these limitations, it is necessary to investigate the relationship between PR3-ANCA and the effects of several induction therapies in a large prospective study across multiple ethnic groups. Despite these limitations, this is the first study to demonstrate that PR3-ANCA can serve as a predictor of non-response to steroid therapy in patients with UC. The results obtained in this study encourage researchers to investigate the mechanism by which PR3ANCA aggravates inflammation in UC.

\section{Conclusions}

Approximately half of active-phase UC cases were positive for serum PR3-ANCA and were predictive of more severe disease as well as failure of steroid therapy. PR3ANCA may exacerbate inflammation of the mucosa in UC, and inhibiting PR3-ANCA may be useful as a strategy for induction therapy. We have launched a prospective study to clarify the relationship between the efficacy of different induction therapies and PR3-ANCA, and the relationship between the relapse of UC and PR3-ANCA.

\begin{abstract}
Abbreviations
ANCA: Antineutrophil cytoplasmic antibody; Cl: Confidence interval; CLEIA: Chemiluminescent enzyme immunoassays; CMV: Cytomegalovirus; CRP: C-reactive protein; EIA: Enzyme immunoassay; ELISA: Enzyme-linked immunosorbent assays; IIF: Indirect immunofluorescence; LCAP: Leukocytapheresis; LRG: Leucine-rich alpha-2 glycoprotein; MES: Mayo Endoscopic Subscore; MPO: Myeloperoxidase; MPO-ANCA: Myeloperoxidase antineutrophil cytoplasmic antibody; NETs: Neutrophil extracellular traps; PR3: Anti-proteinase 3; PR3ANCA: Anti-proteinase 3 antineutrophil cytoplasmic antibody; UC: Ulcerative colitis; 5-ASA: 5-Aminosalicylic acid.
\end{abstract}

\section{Acknowledgements}

We would like to thank Editage (www.editage.com) for English language editing.

\section{Authors' contributions}

Conception and design: TI, ST; Acquisition of data: YA, HY, MW, MA, SN, KM; Analysis and interpretation of data: TM, YA, Tl; Drafting of the manuscript: YA, TI, ST; Revision: SH, TM, HSC; Supervision: HO. All authors have read and approved the final version to be published.

\section{Funding}

None.

\section{Availability of data and materials}

The datasets generated and/or analyzed during the current study are available from the corresponding author upon reasonable request.

\section{Declarations}

Ethics approval and consent to participate

This study was approved by the Ethics Committee of Kagawa Prefectural Central Hospital (No. 861) and the Ethics Committee of Mitoyo General Hospital
(No. 19CR01-101). All procedures performed in studies involving human participants were in accordance with the ethical standards of the institutional and/or national research committee and with the 1964 Helsinki declaration and its later amendments or comparable ethical standards. Administrative permissions were not required to access the raw data of this study. The Ethics Committee of Kagawa Prefectural Central Hospital and the Ethics Committee of Mitoyo General Hospital permit an opt-out method for the agreement of the patients to participate in the study. The requirement for written informed consent to participate in the study was replaced by an opt-out method, owing to the retrospective nature of this study.

\section{Consent for publication}

The requirement for written informed consent was replaced by an opt-out method owing to the retrospective nature of this study.

\section{Competing interests}

The authors have no conflicts of interest to declare.

\section{Author details}

${ }^{1}$ Department of Gastroenterology, Kagawa Prefectural Central Hospital, 1-2-1 Asahi-machi, Takamatsu, Kagawa 760-8557, Japan. ²Department of Gastroenterology, Mitoyo General Hospital, 708 Himehama, Toyohama-cho, Kan-onji, Kagawa 769-1695, Japan. ${ }^{3}$ Department of Gastroenterology and Hepatology, Okayama University Graduate School of Medicine, Dentistry and Pharmaceutical Sciences, 2-5-1 Shikata-cho, Kita-ku, Okayama 700-8558, Japan. ${ }^{4}$ Department of Clinical Epidemiology, Hyogo College of Medicine, 1-1 Mukogawa-cho, Nishinomiya, Hyogo 663-8501, Japan. ${ }^{5}$ Department of Pathology, Kagawa Prefectural Central Hospital, 1-2-1 Asahi-machi, Takamatsu, Kagawa 760-8557, Japan.

Received: 3 January 2021 Accepted: 13 August 2021

Published online: 23 August 2021

\section{References}

1. Ungaro R, Mehandru S, Allen PB, Peyrin-Biroulet L, Colombel JF. Ulcerative colitis. Lancet. 2017;389:1756-70.

2. Faubion WA Jr, Loftus EV Jr, Harmsen WS, Zinsmeister AR, Sandborn WJ. The natural history of corticosteroid therapy for inflammatory bowel disease: a population-based study. Gastroenterology. 2001;121:255-60.

3. Jeon HH, Lee HJ, Jang HW, Yoon JY, Jung YS, Park SJ, et al. Clinical outcomes and predictive factors in oral corticosteroid-refractory active ulcerative colitis. World J Gastroenterol. 2013;19:265-73.

4. Lindgren SC, Flood LM, Kilander AF, Löfberg R, Persson TB, Sjödahl RI. Early predictors of glucocorticosteroid treatment failure in severe and moderately severe attacks of ulcerative colitis. Eur J Gastroenterol Hepatol. 1998;10:831-5

5. Danese S, Fiocchi C. Ulcerative colitis. N Engl J Med. 2011;365:1713-25.

6. Geng X, Biancone L, Dai HH, Lin JJ, Yoshizaki N, Dasgupta A, Pallone F, et al. Tropomyosin isoforms in intestinal mucosa: production of autoantibodies to tropomyosin isoforms in ulcerative colitis. Gastroenterology. 1998;114:912-22.

7. Kuwada T, Shiokawa M, Kodama Y, Ota S, Kakiuchi N, Nannya Y, et al. Identification of an anti-integrin av $\beta 6$ autoantibody in patients with ulcerative colitis. Gastroenterology. 2021;160:2383-94.

8. Bar Yehuda S, Axlerod R, Toker O, Zigman N, Goren I, Mourad V, et al. The association of inflammatory bowel diseases with autoimmune disorders: a report from the epi-IIRN. J Crohns Colitis. 2019;13:324-9.

9. Lüdemann J, Utecht B, Gross WL. Detection and quantitation of antineutrophil cytoplasm antibodies in Wegener's granulomatosis by ELISA using affinity-purified antigen. J Immunol Methods. 1998;114:167-74.

10. Pagnoux C. Updates in ANCA-associated vasculitis. Eur J Rheumatol. 2016;3:122-33

11. Quinton JF, Sendid B, Reumaux D, Duthilleul P, Cortot A, Grandbastien $B$, et al. Anti-saccharomyces cerecisiae mannan antibodies combined with antineutrophil cytoplasmic autoantibodies in inflammatory bowel disease: prevalence and diagnostic role. Gut. 1998;42:788-91.

12. Rump JA, Schölmerich J, Gross V, Roth M, Helfesrieder R, Rautmann A, et al. A new type of perinuclear anti-neutrophil cytoplasmic antibody 
( $p$-ANCA) in active ulcerative colitis but not in Crohn's disease. Immunobiology. 1990;181:406-13.

13. Kovacs M, Lakatos PL, Papp M, Jacobsen S, Nemes E, Polgar M, et al. Pancreatic autoantibodies and autoantibodies against goblet cells in pediatric patients with inflammatory bowel disease. J Pediatr Gastroenterol Nutr. 2012;55:429-35.

14. XU J, Yang CH, Chen XY, Li XH, Dai M, Xiao SD. A subset of ulcerative colitis with positive proteinase-3 antineutrophil cytoplasmic antibody. World J Gastroenterol. 2008;14:7012-5.

15. Takedatsu H, Mitsuyama K, Fukunaga S, Yoshioka S, Yamauchi R, Mori A, et al. Diagnostic and clinical role of serum proteinase 3 antineutrophil cytoplasmic antibodies in inflammatory bowel disease. J Gastroenterol Hepatol. 2018:33:1603-7.

16. Frøslie KF, Jahnsen J, Moum BA, Vatn MH. Mucosal healing in inflammatory bowel disease: results from a Norwegian population-based cohort. Gastroenterology. 2007;133:412-22.

17. Nakarai A, Kato J, Hiraoka S, Kuriyama M, Akita M, Hirakawa T, et al. Evaluation of mucosal healing of ulcerative colitis by a quantitative fecal immunochemical test. Am J Gastroenterol. 2013;108:83-9.

18. Takashima S, Kato J, Hiraoka S, Nakarai A, Takei D, Inokuchi T, et al. Evaluation of mucosal healing in ulcerative colitis by fecal calprotectin vs. fecal immunochemical test. Am J Gastroenterol. 2015;1 10:873-80.

19. Mandl LA, Solomon DH, Smith EL, Lew RA, Katz JN, Shmerling RH. Using antineutrophil cytoplasmic antibody testing to diagnose vasculitis: Can test-ordering guidelines improve diagnostic accuracy? Arch Intern Med. 2002;162:1509-14.

20. Shirai T, Takahashi R, Tajima Y, Kohata K, Yamamoto J, Fujii H, et al. Peripheral T cell lymphoma with a high titer of proteinase-3-antineutrophil cytoplasmic antibodies that resembled Wegener's granulomatosis. Intern Med. 2009;48:2041-5.

21. Lennard-Jones JE. Classification of inflammatory bowel disease. Scand J Gastroenterol. 1989;170:2-6.

22. Satsangi J, Silverberg MS, Vermeire S, Colombel JF. The Montreal classification of inflammatory bowel disease: controversies, consensus, and implications. Gut. 2006;55:749-53.

23. Schroeder KW, Tremaine WJ, IIstrup DM. Coated oral 5-aminosalicylic acid therapy for mildly to moderately active ulcerative colitis. A randomized study. N Engl J Med. 1987;317:1625-9.

24. Matts SG. The value of rectal biopsy in the diagnosis of ulcerative colitis. Q J Med. 1961;30:393-407.

25. Truelove SC, Jewell DP. Intensive intravenous regimen for severe attacks of ulcerative colitis. Lancet. 1974;1:1067-70.

26. Lopez-Sanroman A, Bermejo F, Carrera E, Garcia-Plaza A. Efficacy and safety of thiopurinic immunomodulators (azathioprine and mercaptopurine) in steroid-dependent ulcerative colitis. Aliment Pharmacol Ther. 2004:20:161-6.

27. Vermeire S, Van Assche G, Rutgeerts P. C-reactive protein as a marker for inflammatory bowel disease. Inflamm Bowel Dis. 2004;10:661-5.

28. Srivastava S, Kedia S, Kumar S, Pratap Mouli V, Dhingra R, Sachdev V, et al. Serum human trefoil factor 3 is a biomarker for mucosal healing in ulcerative colitis patients with minimal disease activity. J Crohns Colitis. 2015;9:575-9.

29. Shinzaki S, Matsuoka K, lijima H, Mizuno S, Serada S, Fujimoto M, et al. Leucine-rich Alpha-2 Glycoprotein is a serum biomarker of mucosal healing in ulcerative colitis. J Crohns Colitis. 2017;11:84-91.

30. Naka T, Fujimoto M. LRG is a novel inflammatory marker clinically useful for the evaluation of disease activity in rheumatoid arthritis and inflammatory bowel disease. Immunol Med. 2018;41:62-7.

31. Elzouki AN, Eriksson S, Löfberg R, Nässberger L, Wieslander J, Lindgren S. The prevalence and clinical significance of alpha 1-antitrypsin deficiency
(PiZ) and ANCA specificities (proteinase 3, BPI) in patients with ulcerative colitis. Inflamm Bowel Dis. 1999;5:246-52.

32. García-Herola A, Nos P, Hoyos M, Hinojosa J, Molés JR, Pascual S, et al. Significance of the determination of antineutrophil cytoplasmic antibodies (ANCA) in ulcerative colitis and Crohn's disease. Gastroenterol Hepatol. 1998;21:169-73.

33. Cambridge G, Rampton DS, Stevens TR, McCarthy DA, Kamm M, Leaker B. Anti-neutrophil antibodies in inflammatory bowel disease: prevalence and diagnostic role. Gut. 1992;33:668-74.

34. Arias-Loste MT, Bonilla G, Moraleja I, Mahler M, Mieses MA, Castro B, et al. Presence of anti-proteinase 3 antineutrophil cytoplasmic antibodies (antiPR3 ANCA) as serologic markers in inflammatory bowel disease. Clin Rev Allergy Immunol. 2013;45:109-16.

35. Horn MP, Peter AM, Righini Grunder F, Leichtle AB, Spalinger J, Schibli S, et al. PR3-ANCA and panel diagnostics in pediatric inflammatory bowel disease to distinguish ulcerative colitis from Crohn's disease. PLoS ONE. 2018;13:e0208974. https://doi.org/10.1371/journal.pone.0208974.

36. Mizuochi T, Arai K, Kudo T, Nambu R, Tajiri H, Aomatsu T, et al. Diagnostic accuracy of serum proteinase 3 antineutrophil cytoplasmic antibodies in children with ulcerative colitis. J Gastroenterol Hepatol. 2021:36:1538-44.

37. Stinton LM, Bentow C, Mahler M, Norman GL, Eksteen B, Mason AL, et al. PR3-ANCA: a promising biomarker in primary sclerosing cholangitis (PSC). PLoS ONE. 2014;9:e112877. https://doi.org/10.1371/journal.pone.01128 77.

38. Kessenbrock K, Krumbholz M, Schönermarck U, Back W, Gross WL, Werb Z, et al. Netting neutrophils in autoimmune small-vessel vasculitis. Nat Med. 2009;15:623-5.

39. Nakazawa D, Shida H, Tomaru U, Yoshida M, Nishio S, Atsumi T, et al. Enhanced formation and disordered regulation of NETs in myeloperoxidase-ANCA-associated microscopic polyangiitis. J Am Soc Nephrol. 2014;25:990-7.

40. Brinkmann V, Reichard U, Goosmann C, Fauler B, Uhlemann Y, Weiss DS, et al. Neutrophil extracellular traps kill bacteria. Science. 2004;303:1532-5.

41. Hakkim A, Fürnrohr BG, Amann K, Laube B, Abed UA, Brinkmann V, et al. Impairment of neutrophil extracellular trap degradation is associated with lupus nephritis. Proc Natl Acad Sci USA. 2010;107:9813-8.

42. Döring $Y$, Weber $C$, Soehnlein O. Footprints of neutrophil extracellular traps as predictors of cardiovascular risk. Arterioscler Thromb Vasc Biol. 2013;33:1735-6.

43. Grayson PC, Kaplan MJ. At the Bench: neutrophil extracellular traps (NETs) highlight novel aspects of innate immune system involvement in autoimmune disease. J Leukoc Biol. 2016;99:253-64.

44. Nakazawa D, Tomaru U, Suzuki A, Masuda S, Hasegawa R, Kobayashi T, et al. Abnormal conformation and impaired degradation of propylthiouracil-induced neutrophil extracellular traps: implications of disordered neutrophil extracellular traps in a rat model of myeloperoxidase antineutrophil cytoplasmic antibody-associated vasculitis. Arthritis Rheum. 2012;64:3779-87.

45. Mukhtyar C, Guillevin L, Cid MC, Dasgupta B, de Groot K, Gross W, et al. EULAR recommendations for the management of primary small and medium vessel vasculitis. Ann Rheum Dis. 2009;68:310-7.

46. Lapraik C, Watts R, Bacon P, Carruthers D, Chakravarty K, D'Cruz D, et al. BSR and BHPR quidelines for the management of adults with ANCA associated vasculitis. Rheumatology (Oxford). 2007;46:1615-6.

\section{Publisher's Note}

Springer Nature remains neutral with regard to jurisdictional claims in published maps and institutional affiliations. 